\title{
Research of Ontology based Battlefield Scenario Generation Systems
}

\author{
Dongsu Jeong, Dohyun Kim and Yoonho Seo* \\ Department of Industrial Management of Korea University, College of Engineering 550-ho, Anam-dong 4-ga, Seongbuk-gu, \\ Seoul 136-701, Republic of Korea \\ ${ }^{*}$ Corresponding author
}

\begin{abstract}
In battlefield, it is very difficult to consider all conditions such as various battlefield environments \& factors. Thus, Friendly forces should satisfy battle tactics, weapon systems and other multiple conditions by responding immediately to battlefield situations. Therefore, in order to suggest the most optimal scenario for current situations, our forces maintain current strengths at the time and require research that can propose a tactic to win the war. In this paper, various situations, spasmodic fighting, and objects with specific performances that can happen in battlefield set up the Battlefield Ontology based on METT-TC (BOMT). Hereupon, we suggest creating an Ontologybased Battlefield Scenario Generation System (OBSG System) that can minimize the damage of our forces and make an optimal decision.
\end{abstract}

Keywords-component; battlefield ontology; performance evaluation object; METT-TC; battlefield ontology based on METTTC (BOMT); ontology-based battlefield scenario generation system (OBSG System)

\section{INTRODUCTION}

It is difficult to minimize the damage of our forces from spasmodic fighting \& various situations in battlefield and respond immediately to battlefield situations. It is necessary to suggest an optimal scenario in current situation based on complex conditions representing the battlefield environment and battle tactics, enemy situation and so on with consideration to every situation happening in the battlefield. Advanced counties generated the C4I system (Command, Control, Communication, Computer and Intelligence System) in order to forecast and analyze the battlefield situation. The C4I system helps the decision making when our forces carry out the military tactics in actual military spot [1]. In MAK, VR-Force that is scenario-based process technology has been used for the tactical training, scenario creation, training situation and situation analysis and etc., [2]. Also, the battlefield environment and situation are represented and analyzed through STAGE (Scenario Tactic and Generation Environment), which is the Scenario-based Modeling \& Simulation (SM\&S) tools in eNGENUTY [3]. In addition, various research for representing the battlefield situation have been conducted, and Process-based Method Research which represents the performance of object as a series of procedures has been carried out to present the battlefield situations as procedural aspects [4]. Research creating an optimal path by using an ontology through Factors, which have effect on movement of troops in actual combat has been progressed [5, 6]. In addition, research of ontology-based aware-ness situation modeling business to process situational information efficiently has been carried out [7]. However, research on designing logical mathematic models to minimize damage by anticipating the battlefield has limitations because of the complexity of the battlefield conditions. Thus, the simulation based approach can be a realistic alternative. In this paper, we aim at development of a scenario generation system to generate alternatives at anticipatable battlefield situations.

\section{Ontology BASEd BATtLefield ScEnario SyStem} (OBSG SYSTEM)

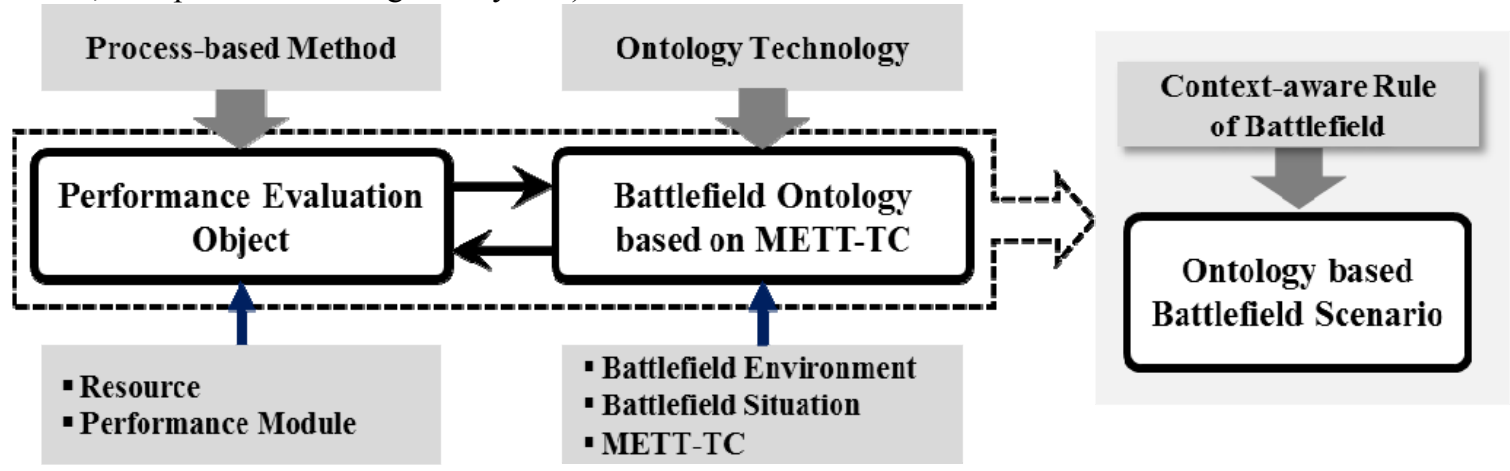

FIGURE I. PROCEDURE OF ONTOLOGY BASED BATTLEFIELD SCENARIO GENERATION SYSTEM

The purpose of this paper is to create diverse scenarios following the training objective by converting battlefield environments and situations to scenario, and to develop the
Ontology-based Battlefield Scenario Generation System (OBSG System) which can counteract the enemy attack in advance. Figure $\mathrm{I}$ is a procedure for presenting the OBSG 
System. First, objects with specific performances in battlefield create logically presented process-based Performance Evaluation Objects. Then, Battlefield Ontology based on METT-TC (BOMT) is created through the METT-TC with diverse situations such as battlefield situations \& tactics and environment based on ontology. Next, the optimal scenario that can perform specific purpose is generated by considering the Process-based Performance Evaluation Objects and complex factors along with battlefield situations \& specific conditions.

\section{A. Process Based Performance Evaluation Object}

In order to win at the battlefield, our forces should accomplish specific purposes, which is to perform each troops $\&$ weapon systems. In addition, it is very important to deduce and represent a complex correlation between interactive elements that are connected complicatedly in weapon systems. Therefore, in order to create a performance evaluation of weapon systems this paper refers to the research of "Processbased modeling for Performance Evaluation of weapon systems". This research can help to select suitable performance objects with requirements of the decision maker and can evaluate the performance of weapon system standing on the basis process [4].
First of all, a standard scenario has to define the creation of evaluating the object. After, a Performance Evaluation Object that can perform specific performances is created through the combination of Resource representing main units of weapon systems and Performance module, which can present specific performances of objects, based on defined scenario. Resource is created by combining the physical component with hardware characters and the behavioral component with dynamic characters. Performance module that can present the function of a particular object is created through process-based method called procedural interworking method by using the basic performance factors, which represent the common function from names of the complex and various functions [8].

Figure II represents the procedure for creation of Performance Evaluation Object. In order to combine $<$ Aircraft $>$ as resource model and <Detect module $>$ as performance module, Performance factors classify the expression of performance module. Next, the Performance object that can perform the Detect with $<$ Aircraft $>$ of Resource model is created through interworking between resource model and performance module. Created Evaluation Objects will perform distinct performance, and can analyze the performance $\&$ effect of weapon object.

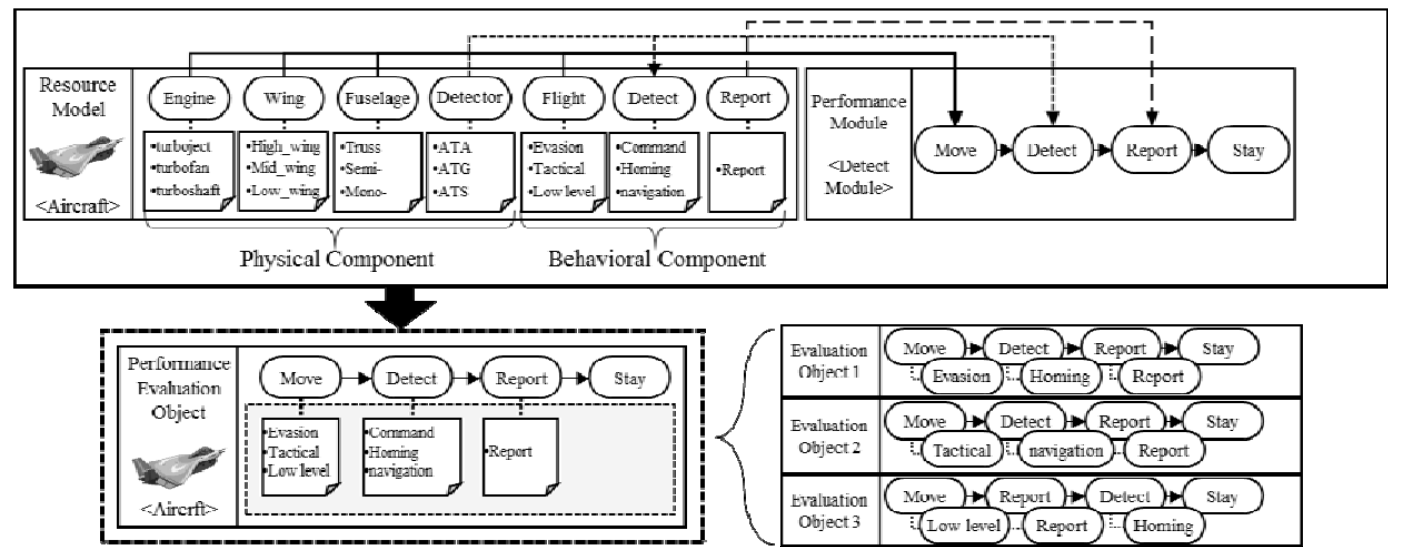

FIGURE II. PERFORMANCE EVALUATION OBJECT CREATION

\section{B. Battlefield Ontology Based on METT-TC}

Situation recognition is essential to develop dynamic scenarios corresponding to battlefield situations. Recognizing the situations is to understand the environmental relationships to generate scenarios, and then the situational information can be used to decide the direction of the development. We have designed a "Battlefield Ontology based on METT-TC (BOMT)" in order to formally and explicitly specify a network that classifies various elements and their inherent relations of each situation. The designed BOMT helps comprehensive understanding of the given situation, and pro-vides information for the judgment of the given situation.

Figure III represents the METT-TC described as vertical and horizontal relationship. To develop a performance object, the given situation is recognized by the battlefield ontology, and the next performance object to generate scenario is derived.
TABLE I. RELATION BETWEEN CLASS AND RELATED CLASS

\begin{tabular}{|c|c|c|}
\hline Class Name & Related Class Name & Relation \\
\hline \multirow{2}{*}{ Troops } & Battlefield & isPartOf \\
\cline { 2 - 3 } & Mission & hasMission \\
\cline { 2 - 3 } & Terrain & areLocatedIn \\
\hline \multirow{2}{*}{ Mission } & Battlefield & isPartOf \\
\cline { 2 - 3 } & Time & isOperatedAt \\
\hline \multirow{2}{*}{ Terrain } & Battlefield & isPartOf \\
\cline { 2 - 3 } & Weather & isPartOf \\
\hline Weather & Battlefield & isPartOf \\
\hline Civil & Battlefield & isPartOf \\
\hline 1Time & Battlefield & isAttackedBy \\
\hline \multirow{2}{*}{ Our force } & Enemy & \\
\cline { 2 - 3 } & Auciliary & Auciliary \\
\hline Enemy & \multicolumn{2}{|c|}{} \\
\hline
\end{tabular}




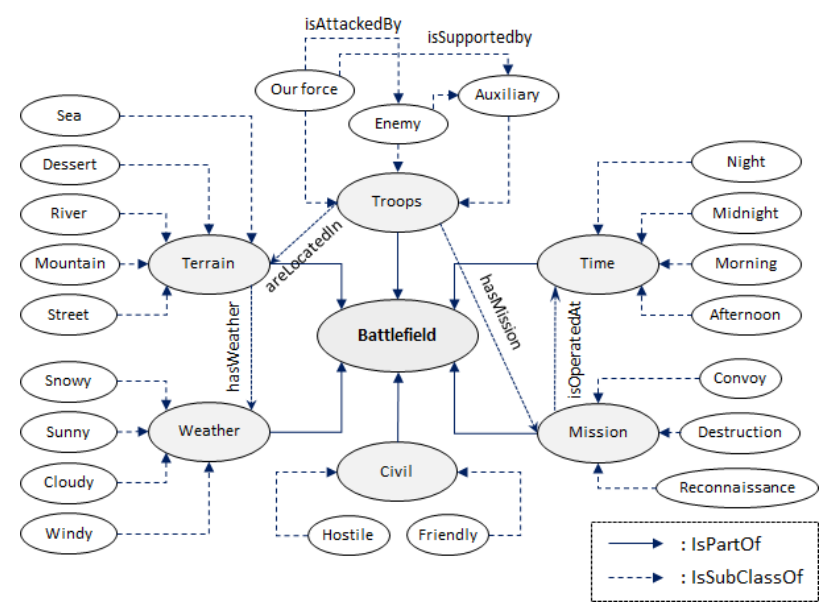

FIGURE III. PERFORMANCE EVALUATION OBJECT CREATION

\section{Ontology Based Battlefield Scenario Generation}

To generate the appropriate scenario for a given battlefield situation, we require scenario generation rules that support the selection of a performance object, which contains the most appropriate performances considering complex elements in a battlefield. The scenario generation rules described below generate the sequence of previously generated performance objects by using the battlefield ontology. In the given battlefield situation, the availability of the troops is judged by terrain and weather information, and then the performance object is derived by checking mission, time and enemy situational information.

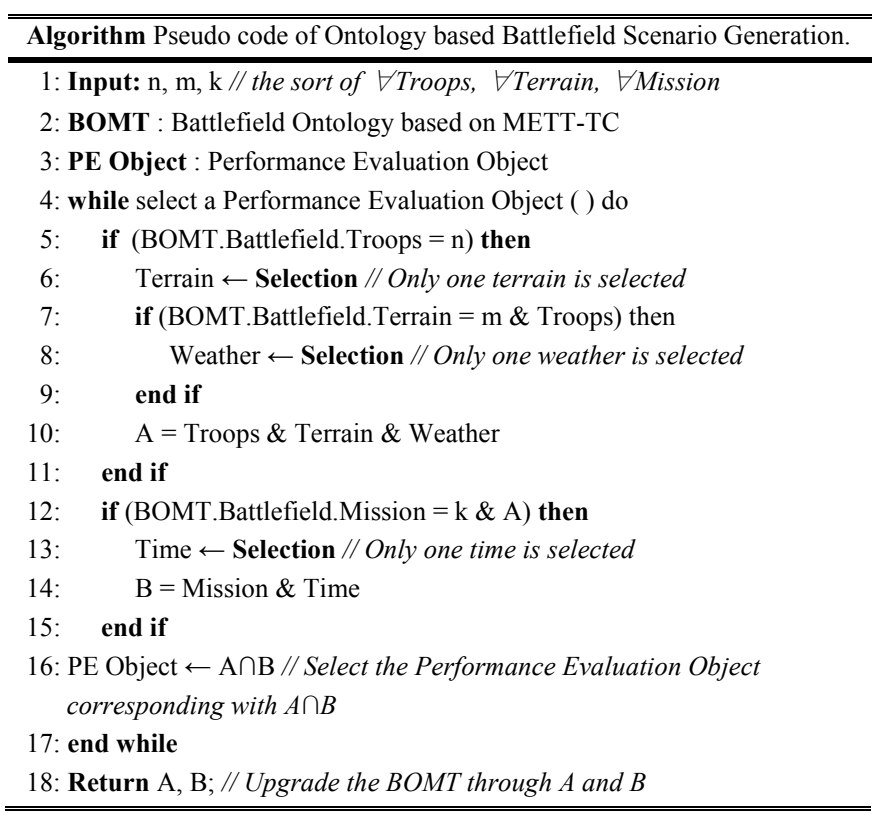

\section{CASE StUdy}

In this section, we suggest an example for selection and development of the performance object by using tactical judgment based on the previously designed battle-field ontology. The example presents recursive process to achieve the purpose, "Destroy an enemy command post". For the first iteration, the scenario generation process derives an "Attack" module after judging availability of our forces' fighter with conditions (terrain, weather and time) in the battlefield ontology, and comparing strength of our forces and enemy. Likewise, for the next iteration the scenario generation process derives the "Attack" module to destroy enemy command post after checking the availability of our forces' fighter. For the last iteration, the scenario generation process derives a "Stay" module after confirming that the purpose is achieved.

TABLE II. EXAMPLE OF CASE STUDY

\begin{tabular}{|c|c|}
\hline Purpose & Destroy the enemy command post and aggressor forces \\
\hline $\begin{array}{l}\text { Iteration(1) } \\
\text { : "Attack" } \\
\text { Module }\end{array}$ & $\begin{array}{c}\text { Step } 1: \text { isLocatedIn(Fighter, Mountain) and hasWeather } \\
\text { (Mountain, Sunny) } \rightarrow \text { (Module }<- \text { "Move") } \\
\text { Step } 2: \text { (Module=="Move") and isAttackedBy(Fighter, } \\
\text { Enemy_Fighter)and(Fighter.Strength }> \\
\text { Enemy_Fighter.Strength) and isOperatedAt(Destruction, } \\
\text { Morning) } \rightarrow \text { (Module }<- \text { "Attack") }\end{array}$ \\
\hline $\begin{array}{l}\text { Iteration(2) } \\
\text { : "Attack" } \\
\text { Module }\end{array}$ & $\begin{array}{c}\text { Step 1: isLocatedIn(Fighter, Mountain) and hasWeather } \\
\text { (Mountain, Sunny) } \rightarrow \text { (Module }<- \text { "Move") } \\
\text { Step 2: (Module=="Move") and isAttackedBy(Fighter, } \\
\text { Enemy_Command post) and (Fighter.Strength }> \\
\text { Enemy_Command post.Strength) } \\
\text { and isOperatedAt(Destruction, Morning) } \rightarrow \text { (Module }<- \\
\text { "Attack") }\end{array}$ \\
\hline $\begin{array}{l}\text { Iteration(3) } \\
\text { : "Stay" } \\
\text { Module }\end{array}$ & $\begin{array}{c}\text { Step 1: isLocatedIn(Fighter, Mountain) and hasWeather } \\
\text { (Mountain, Sunny) } \rightarrow \text { (Module }<- \text { "Move") } \\
\text { Step 2: Achievement of goal } \rightarrow \text { (Module }<- \text { "Stay") }\end{array}$ \\
\hline
\end{tabular}

\section{CONCLUSION}

The judgment based on situational recognition is essential in the scenario generation process. In this paper, the battlefield ontology based on the METT-TC (BOMT) can specify the comprehensive relationships among the related information for battlefield, and can suggest the foundation of judging for the development of a scenario. In brief, this study designed various elements and their relationships as the battlefield ontology, and applied them to generate the scenario. Consequently, the system pro-posed in this study can support the logical development of the performance object and provide dynamic scenario generation based on BOMT, which reflects the results of the previously developed performance object.

\section{ACKNOWLEDGMENT}

This work was supported by Defense Acquisition Program Administration and Agency for Defense Development under the contract UD110006MD and UD140022PD, Korea.

\section{REFERENCES}

[1] Salmon, P., Stanton, N., Walker, G., \& Green, D. "Situation awareness measurement: A review of applicability for C4I environments," Applied ergonomics, Vol. 37, No. 2, pp.225-238, 2006

[2] Fern, L., \& Shively, R. J, “A comparison of varying levels of automation on the supervisory control of multiple UASs," Proceedings of AUVSI's Unmanned Systems North America 2009, pp.10-13, 2009

[3] Ahn, E., Chang, D. S., Pyun, J., Kwon, Y. J., \& Park, S. C., "Development of Timely Counter-scenario on Small Scale 
Engagements," Journal of the Korea Society for Simulation, Vol. 22, NO. 2, pp.63-71, 2013

[4] Jeong, D., Seo, Y., "Process-based Modeling for Performance Evaluation of Weapon Systems," The 15th Asia Simulation Conference 2015, Vol. 8, 2015

[5] Ma, Y. B., Kim, J. K., \& Lee, J. S., "Modeling and simulation of ontology-based path finding in war-game simulation," Journal of the Korea Society for Simulation, Vol. 21, No.1, pp.9-17, 2012

[6] Ahmed, R., Shah, M., Umar, M., "CONCEPTS OF SIMULATION MODEL SIZE AND COMPLEXITY," International Journal of simulation modelling, Vol. 15, No. 2, pp.213-222, 2016

[7] Kim, J. D., Son, J.,Kim, J., Baik, D. K., "A Modeling Method of Context-Aware based on Ontology," Korea Information Science Society, Vol 38, pp.65-68, 2011

[8] Oh, S., Jeong, D., Seo, Y., "Scenario-based Evaluation Object Selection Research for Performance Evaluation of Weapon," Proceedings of Joint Conference Proceedings Industrial Engineering. pp.1239-1244, 2016

[9] Ulicny, B., Matheus, C., Powell, G., Dionne, R., Kokar, M., "Representability of METT-TC factors in JC3IEDM. DTIC Document," 2007 